\title{
Analytical Matched Filter Bound for $M$-QAM Hierarchical Constellations with Diversity Reception in Multipath Rayleigh Fading Channels
}

\author{
Nuno Souto, Rui Dinis, and João Carlos Silva
}

\begin{abstract}
In this paper, we derive matched filter bound (MFB) expressions for the performance of hierarchical quadrature amplitude modulations ( $M$-HQAM) over time-discrete multipath Rayleigh fading environments with diversity. The expressions are valid for multipath environments where different paths and antennas can be correlated. Since hierarchical constellations are usually employed to achieve unequal bit error protection, the MFB expressions are given in terms of the individual bit error rate (BER) for the different bit streams. Although typically the MFB is obtained assuming slow time varying channels, fast varying channels are also considered in this paper.
\end{abstract}

Index Terms-Matched filter bound, diversity, hierarchical constellations, quadrature amplitude modulation, Rayleigh fading.

\section{INTRODUCTION}

W HEN supporting broadcast transmissions in wireless communication networks it is possible to exchange some of the capacity of the good communication links to the poor ones and this tradeoff can be worthwhile as was shown in [1]. Hierarchical quadrature amplitude modulations ( $M$-HQAM) constitute a very simple method to provide unequal bit error protection and improve the efficiency of a network when broadcasting information to several users. If the information is scalable (coded voice or video signals [1], [2]), several data streams can be transmitted simultaneously with different error protection levels and, depending on the propagation conditions, a given user can attempt to demodulate only the more protected bits or also the less protected bits carrying additional information. 16-HQAM and 64-HQAM constellations have already been incorporated into DVB-T standards [3].

Due to the difficulty in dealing with multipath channels, instead of trying to obtain exact performance expressions for these environments it can be satisfactory to just have some analytical expressions representing an ideal performance that may not be possible to achieve in practice but can work as a very important benchmark for the evaluation of a specific receiver. The matched filter bound (MFB) is found assuming perfect channel knowledge and the transmission of a single pulse, i.e., no intersymbol interference (ISI) occurs, and can be regarded as a lower limit on the BER for a particular communication channel. Recently there was as a renewed interest in

Paper approved by M.-S. Alouini, the Editor for Modulation and Diversity Systems of the IEEE Communications Society. Manuscript received September 16, 2008; revised April 2, 2009 and July 31, 2009.

This paper was presented in part at IEEE VTC'09-Fall, Anchorage, USA, September 2009

The authors are with ISCTE/Instituto de Telecomunicações, Av Forças Armadas, Ed. ISCTE, 1649-026, Lisbon, Portugal (e-mail: nuno.souto@1x.it.pt; rdinis@ist.utl.pt; joao.carlos.silva@1x.it.pt).

Digital Object Identifier 10.1109/TCOMM.2010.03.080480 the computation of the MFB since the performance in severely time-dispersive channels with rich multipath propagation can be close to the MFB, provided that suitable equalization schemes are employed combined with diversity techniques. An example is the IB-DFE (Iterative Block Decision Feedback Equalizer) receiver [4], [5], which is a promising iterative frequency-domain equalization for cyclic-prefix-assisted block transmission schemes. MFB expressions for BPSK and QPSK transmission over multipath Rayleigh fading channels were obtained in [6]-[9] and were extended to QAM in [10]. In this paper we derive bounds for the BERs of the different types of bits (in terms of error protection level) in $M$-HQAM constellations. The presented expressions are valid when we have receive diversity and time-discrete multipath Rayleigh fading channels, where the different paths and receive antennas can be correlated. While references [6]-[10] assumed slowly time varying channels (approximately constant channel during pulse duration period) in this paper we also deal with the more general case where this restriction is not applied.

The paper is organized as follows. Section II describes the model of the communication system and gives a brief description of $M$-HQAM constellations. Section III derives MFB BER expressions for slow and fast fading channels and Section IV presents and analyzes some numerical results. The conclusions are given on Section V.

\section{System And Channel Model}

\section{A. Hierarchical QAM Signal Constellations}

In $M$-HQAM constellations it is possible to have $1 / 2-$ $\log _{2} M$ classes of bits with different error protection and to which different streams of information can be mapped. By using non uniformly spaced signal points it is possible to modify the different error protection levels. $M$-HQAM constellations can be characterized by the ratios of the distances between the inner nested constellations [11]

$$
k_{i}=\frac{D_{i}}{D_{i+1}}, i=1, \ldots, \log _{2} M 2-1
$$

Assuming a normalized constellation (with average symbol energy $E_{s}=1$ ) it is easy to verify that the inner distances can be obtained as

$$
\left\{\begin{array}{c}
D_{\log _{2} M / 2}=\sqrt{\frac{2}{1+\sum_{l=1}^{\log _{2} M 2-1} \prod_{j=1}^{l} k_{\log _{2} M 2-j}^{2}}} \\
D_{i}=D_{\log _{2} M / 2} \prod_{j=1}^{\log _{2} M 2-1} k_{j}, i<\log _{2} M / 2
\end{array}\right.
$$

In our analysis we assume that the parallel information streams are split in two, so that half of each stream goes for the in-phase branch and the other for the quadrature branch of the 
modulator. The resulting bit sequence in each branch is Gray encoded and mapped to the respective $\sqrt{M}$-PAM constellation symbols. The symbols from the in-phase and quadrature branches are then grouped together forming complex $M$-QAM symbols. The Gray coding for each $\sqrt{M}$-PAM constellation is performed according to the procedure described in [12] so that the correspondence between information bits $b_{j}^{i}$ and coded bits $g_{j}^{i}$ is the same as the one presented in that paper.

\section{B. Channel Model}

Let us consider the case of a transmission over an $N_{r x}$ diversity branch multipath Rayleigh fading channel where different branches can have different fading powers. The corresponding transmit/receive block diagram is shown in Fig. 1. Assuming a discrete multipath channel for each diversity branch $n$ composed of $L_{n}$ correlated discrete taps where the magnitude of each tap $i$ has a mean square value of $\Omega_{i, n}^{2}$, the respective response at time $t$ to an impulse applied at $t-\tau$ can be modelled as

$$
c_{n}(\tau, t)=\sum_{i=1}^{L_{n}} \alpha_{i, n}(t) \delta\left(\tau-\tau_{i, n}\right), n=1 \ldots N_{r x},
$$

where $\alpha_{i, n}(t)$ is a zero-mean complex Gaussian random process, $\tau_{i, n}$ is the respective tap delay (assumed constant) and $\delta(t)$ is the Dirac function. Each channel tap autocovariance function can be expressed as [13]

$$
\begin{aligned}
R_{\alpha_{i, n}}(\tau) & =E\left\{\alpha_{i, n}(t) \alpha_{i, n}(t+\tau)\right\} \\
& =\Omega_{i, n}^{2} J_{0}\left(2 \pi f_{D} \tau\right),
\end{aligned}
$$

where $J_{0}(\cdot)$ is the zeroth-order Bessel function of the first kind and $f_{D}$ is the Doppler frequency. Regarding the crosscorrelation between different taps belonging to the same or to different diversity branches, no restriction is imposed, i.e., all the taps can be correlated.

\section{Matched Filter Bound Derivation}

\section{A. Slow Fading Channel}

For the derivation of the MFB we assume the transmission of only one pulse $s \cdot g(t)$, where $s$ is an HQAM symbol and $g(t)$ is the impulse response of the transmit filter. Although $g(t)$ can have unlimited duration typically it becomes almost zero outside a narrow interval, usually related to the symbol period. If the channel is slowly time-varying it can be considered approximately constant inside this interval and the sampled output after the matched filters, $h_{n}(t)$, and the combiner becomes

$$
y\left(t=t_{0}\right)=s \cdot \sum_{n=1}^{N_{r x}} \sum_{i=1}^{L_{n}} \sum_{i^{\prime}=1}^{L_{n}} \alpha_{i, n} \alpha_{i^{\prime}, n}^{*} R\left(\tau_{i, n} \tau_{i^{\prime}, n}\right)+\sum_{n=1}^{N_{r x}} w_{n},
$$

where $t_{0}$ is the sampling instant, $R(\tau)$ is the autocorrelation function of the transmit filter and $w_{n}$ represents Additive White Gaussian Noise (AWGN) samples with power spectral density $N_{0}$. The instantaneous received signal to noise power ratio is given by $S N R=\frac{E_{s}}{N_{0}} \kappa$, where $E_{s}$ is the average symbol energy and

$$
\kappa=\sum_{n=1}^{N_{r x}} \sum_{i=1}^{L_{n}} \sum_{i^{\prime}=1}^{L_{n}} \alpha_{i, n} \alpha_{i^{\prime}, n}^{*} R\left(\tau_{i, n} \tau_{i^{\prime}, n}\right)=\mathbf{z}^{H} \sum \mathbf{z} .
$$

The last equality in (6) is the matrix representation of $\kappa$ with z being a $L_{\text {total }} \times 1$ (with $L_{\text {total }}=\sum_{n=1}^{N_{r x}} L_{n}$ ) vector containing the random variables $\alpha_{i, n},{ }^{H}$ denoting the conjugate transpose and $\sum$ being a $L_{\text {total }} \times L_{\text {total }}$ Hermitian matrix constructed as a diagonal matrix composed of smaller $L_{n} \times L_{n}$ matrices $\sum_{n}$ whose elements are $\sum_{n}\left(i^{\prime}, i\right)=R\left(\tau_{i, n}-\tau_{i^{\prime}, n}\right)$.

For obtaining the MFB it is necessary to know the probability density function (PDF) of $\kappa$. In [9] it was shown that (6) can be rewritten as

$$
\kappa=\sum_{i=1}^{L_{\text {total }}} \lambda_{i}\left|z_{i}^{\prime}\right|^{2}
$$

where $z^{\prime}$ are uncorrelated unit-variance complex Gaussian variables and $\lambda_{i}$ are the eigenvalues $\left(i=1, . ., L_{\text {total }}\right)$ of matrix $\sum^{\prime}$ which is defined as

$$
\sum^{\prime}=\mathbf{Q}^{H} \sum \mathbf{Q} .
$$

In (8) matrix $\mathbf{Q}$ is obtained from the decomposition of the covariance matrix of $\mathbf{z}$ as $\operatorname{Cov}[\mathbf{z}]=\mathbf{Q Q}^{H}$ (using for example the Cholesky decomposition).

According to (7), $\kappa$ can be expressed as a sum of independent random variables with exponential distributions. We can then apply the characteristic function method combined with partial fractions decomposition to obtain the PDF of $\kappa$. If there are $L^{\prime}$ distinct eigenvalues each with a multiplicity of $\theta_{i},\left(i=1 \ldots L^{\prime}\right)$, the following expression results for the PDF of $\kappa$ :

$$
p(\kappa)=\sum_{i=1}^{L^{\prime}} \sum_{k=1}^{\theta_{i}} \frac{A_{i, k}}{\lambda_{i}^{\theta_{i}}\left(\theta_{i}-k\right) !(k-1) !} \kappa^{k-1} e^{\frac{\kappa}{\lambda_{i}}},
$$

with

$$
A_{i, k}=\left[\frac{\partial^{\theta_{i}-k}}{\partial s^{\theta_{i}-k}}\left(\prod_{\substack{j=1 \\ j \neq 1}}^{L^{\prime}} \frac{1}{\left(1+s \lambda_{j}\right)^{\theta_{j}}}\right)\right]_{s=\frac{1}{\theta_{i}}}
$$

Based on [14, eq.(10)] and using the constellation distance definitions provided in Section II.A (whose symbol energy is now multiplied by scale factor $\kappa$ ) we can write the individual BERs conditioned on $\kappa$ as (11), see top of next page, where we get (12), also at the top of the next page, and

$$
\mathbf{D}_{s}(j)=\sum_{i=1}^{1 / 2 \log _{2} M}\left(2 b_{j}^{i}-1\right) \frac{D_{1 / 2 \log _{2} M-i+1}}{2}
$$

where $\operatorname{erfc}(x)$ is the complementary error function. Averaging (11) over the PDF (9) and taking into account that

$$
\begin{aligned}
& \int_{0}^{+\infty} \frac{1}{2} \operatorname{erfc}(\sqrt{x}) \frac{x^{L-1}}{(L-1) ! \bar{x}_{i}^{L}} e^{-\frac{x}{x_{i}}} d x \\
= & {\left[\frac{1-\mu}{2}\right]^{L} \sum_{r=0}^{L-1}\left(\left(\begin{array}{c}
L-1+r \\
r
\end{array}\right)\right)\left[\frac{1+\mu^{r}}{2}\right] }
\end{aligned}
$$




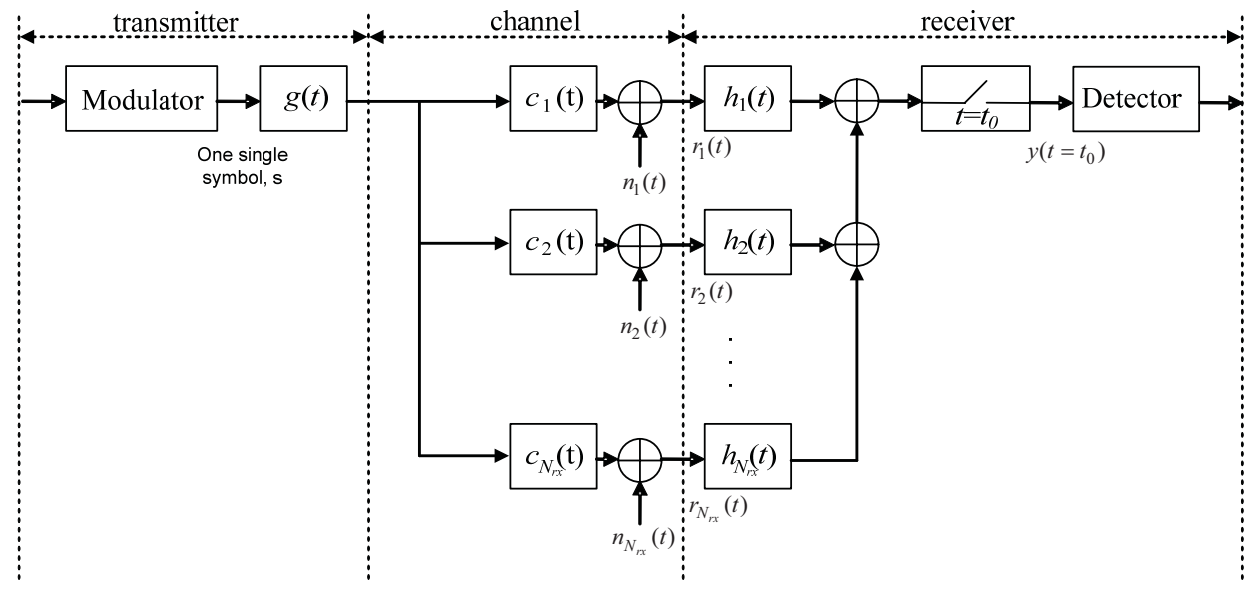

Fig. 1. Baseband transmit/receive scheme.

$$
P_{b}\left(b_{m} \mid \kappa\right)=\frac{2}{\sqrt{M}} \sum_{j=1}^{\sqrt{M} / 2}\left[g_{j}^{m}+(-1)^{g_{j}^{m}} \sum_{l=1}^{2^{(m-1)}}\left[\frac{1}{2}(-1)^{l+1} \times \operatorname{erfc}\left(\sqrt{\frac{E_{s}}{N 0} \kappa}\left[\mathbf{B}_{m}(l)-\mathbf{D}_{s}(j)\right]\right)\right]\right], m=1, \ldots, \log _{2} M / 2
$$

$$
\mathbf{B}_{m}(l)=\frac{\mathbf{D}_{s}\left((2 l-1) 2^{1 / 2 \log _{2} M-m}\right)+\mathbf{D}_{s}\left((2 l-1) 2^{1 / 2 \log _{2} M-m}+1\right)}{2}
$$

$$
\begin{aligned}
P_{b}\left(b_{m}\right)= & \frac{2}{\sqrt{M}} \sum_{j=1}^{\sqrt{M} / 2}\left[g_{j}^{m}+(-1)^{g_{j}^{m}} \sum_{l=1}^{2^{(m-1)}} \frac{1}{2}(-1)^{l+1} \times\right. \\
& \left\{1-\operatorname{sign}\left(\mathbf{B}_{m}(l)-\mathbf{D}_{s}(j)\right)+2 \cdot(-1)^{1 / 2-\operatorname{sign}\left(\mathbf{B}_{m}(l)-\mathbf{D}_{s}(j)\right) / 2} \times\right. \\
& \left.\left.\sum_{i=1}^{L^{\prime}} \sum_{k=1}^{\theta_{i}} \frac{A_{i, k}}{\lambda^{\theta_{i}-k}\left(\theta_{i}-k\right) !}\left[\frac{1-\mu_{i}}{2}\right]^{k} \sum_{r=0}^{k-1}\left(\left(\begin{array}{c}
k-1+r \\
r
\end{array}\right)\right)\left[\frac{1-\mu_{i}}{2}\right]^{r}\right\}\right]
\end{aligned}
$$

with $\mu=\sqrt{\bar{x}_{i} /\left(1+\bar{x}_{i}\right)}$ (see [15]), we can write the individual average BERs as (14), see next page, where

$$
\mu_{i}=\sqrt{\frac{\frac{E_{s}}{N_{0}}\left[\mathbf{B}_{m}(l)-\mathbf{D}_{s}(j)\right]^{2} \lambda_{i}}{1+\frac{E_{s}}{N_{0}}\left[\mathbf{B}_{m}(l)-\mathbf{D}_{s}(j)\right]^{2} \lambda_{i}}} .
$$

and $\operatorname{sign}(x)$ is the sign function (i.e., $\operatorname{sign}(x)=-1$ for $x<0$, 0 for $x=0$ and 1 for $x>0$ ).

\section{B. Fast Fading Channel}

If the channel is too fast to be considered constant during the approximate pulse duration $\left(f_{N}>0.01\right.$, with $f_{N}$ being the normalized Doppler rate defined as $f_{N}=f_{D} T_{s}$, where $T_{s}$ is the symbol period) then a different approach has to be employed for deriving the MFB since (5) will not be a valid representation of the output of the matched filters. To deal with the output of the matched filter we can split the convolution of the filter with the received signal into a sum of $N$ smaller integrals so that the channel can be seen as almost constant inside the resulting shorter time intervals
$(\Delta t)$. Considering the existence of a single diversity branch $\left(N_{r x}=1\right)$ (the extension to multiple diversity branches is straightforward), the sampled output becomes (a somewhat similar approach was also employed in [16] for simplifying a correlation integral)

$$
\begin{array}{r}
y\left(t-t_{0}\right) \approx s \cdot \sum_{i=1}^{L} \sum_{i^{\prime}=1}^{L} \sum_{l=-N / 2}^{N / 2-1} \alpha_{i}(\Delta t / 2+l \Delta t) \\
\cdot \alpha_{i^{\prime}}^{*}(\Delta t / 2+l \Delta t) \\
\cdot R\left(\tau_{i}-\tau_{i \prime}, / \Delta t-\tau_{i}(l+1) \Delta t-\tau_{i}\right)+w
\end{array}
$$

where $R\left(\tau, t_{\text {lower }}, t_{\text {upper }}\right)=\int_{t_{\text {lower }}}^{t_{\text {upper }}} g\left(\tau^{\prime}\right) g^{*}\left(\tau^{\prime}+\tau\right) d \tau$, i.e., it is the partial autocorrelation function of the transmit filter. The total number of intervals $N$ is chosen so that $g(t) \approx 0$ for $|t|>N / 2 \cdot \Delta t$. Variable $\kappa$ is still given by (6) but the 


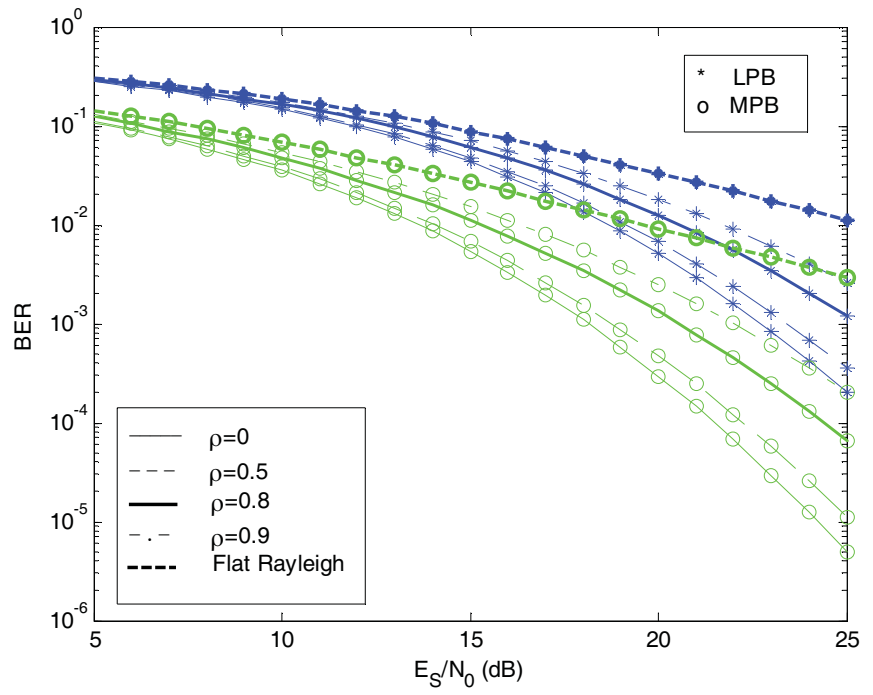

Fig. 2. Impact of correlated taps on the BER of a 16-HQAM constellation with $k_{1}=0.4$ in Vehicular A environment.

elements in $\mathbf{z}$ and $\sum$ are different. The former is defined as

$$
\mathbf{z}=\left[\begin{array}{c}
\alpha_{1}(\Delta t / 2-N / 2 \Delta t) \\
\vdots \\
\alpha_{L}(\Delta t / 2-N / 2 \Delta t) \\
\vdots \\
\alpha_{1}(\Delta t / 2+(N / 2-1) \Delta t) \\
\vdots \\
\alpha_{L}(\Delta t / 2+(N / 2-1) \Delta t)
\end{array}\right] .
$$

Even if the different paths are uncorrelated some of the elements of $\mathbf{z}$ are always correlated, namely the ones belonging to the same taps, due to the inherent time correlation of the channel but we can apply the method of the previous section to make all the components of $\mathbf{z}$ uncorrelated. As for matrix $\sum$ it is still Hermitian and is built as a diagonal matrix composed of smaller $L \times L$ matrices $\sum_{l}(l=-N / 2, \ldots, N / 2-1)$, whose elements are $\sum_{l}\left(i^{\prime}, i\right)=R\left(\tau_{i}-\tau_{i \prime}, l \Delta t-\tau_{i},(l+1) \Delta t-\tau_{i}\right)$. The subsequent derivation and final BER expressions are the same as the ones presented in the previous section.

\section{NumericAl RESUlts}

Using the derived MFB expressions we evaluated the BER of several HQAM constellations in different conditions. In this study we considered the use of a root-square raised cosine (RRC) filter with a roll-off bandwidth factor $\beta=0.22$. The results obtained are plotted as a function of $E_{S} / N_{0}$. Fig. 2 evaluates the effect of the correlation coefficient between taps, $\rho=E\left[\alpha_{i} \alpha_{i^{\prime}}^{*}\right] / \sqrt{E\left[\left|\alpha_{i}\right|^{2}\right] E\left[\left|\alpha_{i^{\prime}}\right|^{2}\right]}$, assumed the same for all the taps, on the performance of a 16-HQAM constellation with $k_{1}=0.4$. The environment considered is the Vehicular A (from [17]). As a reference the results for flat Rayleigh fading are shown in the same graph. Two groups of curves are shown: one for the most protected bits (MPB) and the other for the least protected bits (LPB). As expected, as the correlation increases, the performance degrades due to a loss of the diversity effect from the usage of the different taps.

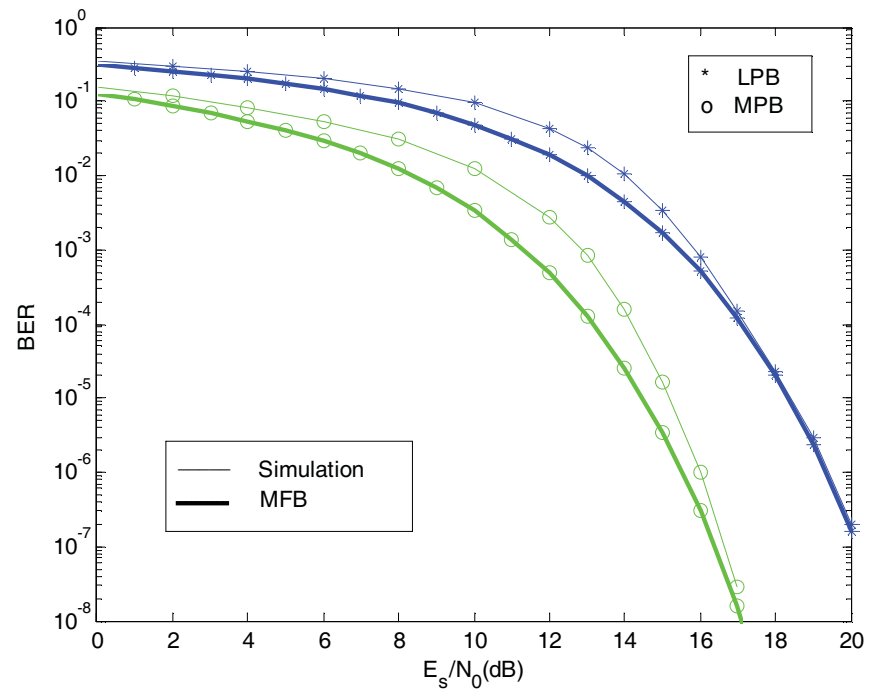

Fig. 3. Simulation versus MFB results for a 16-HQAM constellation with $k_{1}=0.4$ in a 64 taps environment with 2 receiver antennas.

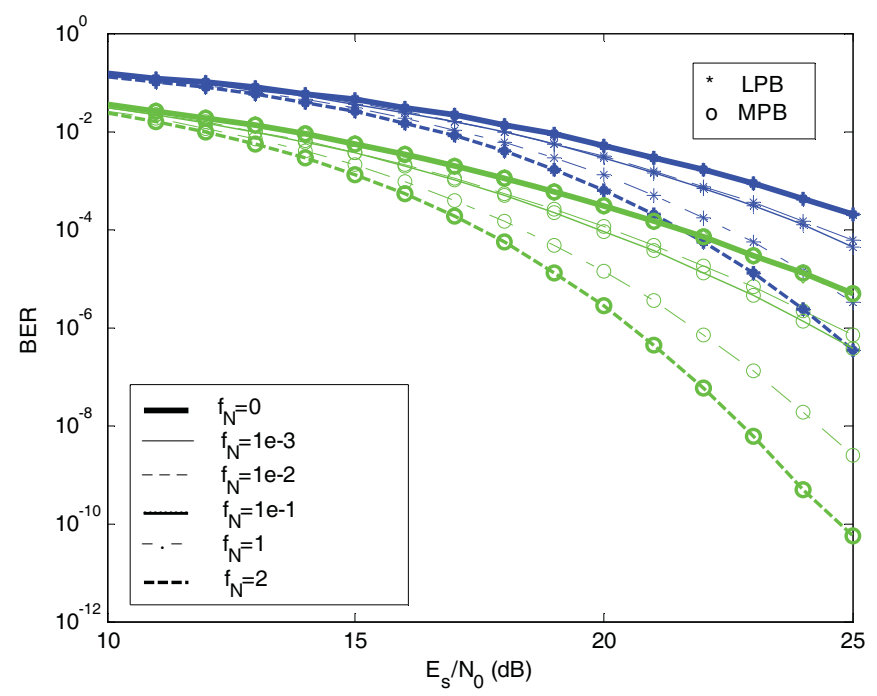

Fig. 4. Impact of the normalized Doppler rate on the BER of a 16-HQAM constellation with $k_{1}=0.4$ in Vehicular A environment.

Nevertheless, this degradation is small until $\rho=0.5$ and even for higher correlation values, like 0.9 , the performance is still relatively far from the flat Rayleigh environment (which does not have any diversity gain).

Fig. 3 compares the MFB with the performance when an IB-DFE receiver [5] is employed. We consider a 16-HQAM constellation with $k_{1}=0.4$ and a channel with 64 equal-power taps and 2 receive antennas. It is clear that the IB-DFE results are asymptotically close to the MFB.

Finally we wanted to evaluate the impact of the channel velocity on the performance of the constellations. To accomplish this it is necessary to employ the approximate MFB expressions described in Section III.B. Fig. 4 presents the results for several normalized Doppler rates, $f_{N}$. The constellation is a 16-HQAM with $k_{1}=0.4$ and the environment is Vehicular A with a Jakes spectrum assumed for the taps. The size of each partition interval, $\Delta t$, is chosen so as to guarantee that $f_{D} \Delta t<0.01$. Looking at the different curves we observe that 
the MFB performance of both bits start to improve substantially as the channel becomes faster. This is a consequence of the fact that, theoretically, in fast fading channels, each individual bit can obtain some diversity gain even without multipath. Although this might suggest that increasing the mobility of the user (or alternatively increasing the symbol period) automatically improves the BER, in practice for very high speeds the channel estimation is extremely difficult and these bounds become unattainable.

\section{CONCLUSiOnS}

In this paper we have derived MFB BER expressions for the individual bit classes of any hierarchical square M-QAM constellation in multipath Rayleigh fading environments with diversity reception. Expressions were obtained for slowly time varying channels as well as for fast varying ones and are valid for environments with correlated taps and for correlated diversity branches. Although MFBs have a deep theoretical nature, they are very important tools for several applications such as gauging the performance of a communication system or to provide a simple validation for efficient channel simulation techniques.

\section{ACKNOWLEDGMENT}

This work was partially supported by the FCT - Fundação para a Ciência e Tecnologia (pluriannual funding and U-BOAT project PTDC/EEA-TEL/67066/2006).

\section{REFERENCES}

[1] T. Cover, "Broadcast channels," IEEE Trans. Inf. Theory, vol. 18, pp. 2-14, Jan. 1972

[2] K. Ramchandran, A. Ortega, K. M. Uz, and M. Vetterli, "Multiresolution broadcast for digital HDTV using joint source/channel coding," IEEE J. Sel. Areas Commun., vol. 11, Jan. 1993.
[3] "Digital video broadcasting (DVB) framing structure, channel coding and modulation for digital terrestrial television (DVB-T) V1.5.1," Nov. 2004, ETSI, European Telecommunication Standard ETS 300744.

[4] N. Benvenuto and S. Tomasin, "Block iterative DFE for single carrier modulation," IEEE Electron. Lett., vol. 39, no. 19, Sep. 2002.

[5] R. Dinis, A. Gusmão, and N. Esteves, "Iterative block-DFE techniques for single-carrier-based broadband communications with transmit/receive space diversity," in Proc. IEEE ISWCS, Sep. 2004.

[6] J. E. Mazo, "Exact matched filter bound for two-beam Rayleigh fading," IEEE Trans. Commun., vol. 49, pp. 1027-1030, July 1991.

[7] M. V. Clark, L. J. Greenstein, W. K. Kennedy, and M. Shafi, "Matched filter performance bounds for diversity combining receivers in digital mobile radio," IEEE Trans. Veh. Technol., vol. 41, pp. 356-362, Nov. 1992.

[8] V. Kaasila and A. Mäammelä, "Bit error probability of a matched filter in a Rayleigh fading multipath channel," IEEE Trans. Commun., vol. 42, pp. 826-828, Feb./Mar./Apr. 1994.

[9] F. Ling, "Matched filter-bound for time-discrete multipath Rayleigh fading channels," IEEE Trans. Commun., vol. 43, pp. 710-713, Feb./Mar./Apr. 1995.

[10] C.-J. Kim, Y.-S Kim, G.-Y. Jeong, and D.-D. Lee, "Matched filter bound of square QAM in multipath Rayleigh fading channels," IEEE Electron. Lett., vol. 33, pp. 20-21, Jan. 1997.

[11] N. Souto, F. Cercas, R. Dinis, and J. C. Silva, "On the BER performance of hierarchical $M$-QAM constellations with diversity and imperfect channel estimation," IEEE Trans. Commun., vol. 55, no. 10, pp. 18521856, Oct. 2007.

[12] P. K. Vitthaladevuni and M.-S. Alouini, "A recursive algorithm for the exact BER computation of generalized hierarchical QAM constellations," IEEE Trans. Inf. Theory, vol. 49, pp. 297-307, Jan. 2003.

[13] G. L. Stüber, Principles of Mobile Communication. Norwell, MA: Kluwer, 2001.

[14] P. K. Vitthaladevuni and M.-S. Alouini, "A closed-form expression for the exact BER of generalized PAM and QAM constelations," IEEE Trans. Commun., vol. 52, pp. 698-700, May 2004.

[15] J. G. Proakis, Digital Communications, 4th ed. McGraw-Hill, 2001.

[16] W. Burchill and C. Leung, "Matched filter bound for OFDM on Rayleigh fading channels," IEEE Electron. Lett., vol. 31, pp. 1716-1717, Sep. 1995.

[17] "Selection procedures for the choice of radio transmission technologies of UMTS," ETSI, TR 101112 v3.2.0, Sophia Antipolis, France, 1998. 